\title{
Inflammation assessment in patients with arthritis using a novel in vivo fluorescence optical imaging technology
}

\author{
Stephanie G Werner, ${ }^{1}$ Hans-Eckhard Langer, ${ }^{1}$ Sarah Ohrndorf, ${ }^{2}$ Malte Bahner, ${ }^{3}$ \\ Peter Schott, ${ }^{4}$ Carsten Schwenke, ${ }^{5}$ Michael Schirner, ${ }^{3}$ Hans Bastian, ${ }^{2}$ \\ Gudrun Lind-Albrecht, ${ }^{1}$ Bernward Kurtz, ${ }^{4}$ Gerd R Burmester, ${ }^{2}$ Marina Backhaus ${ }^{2}$
}

\begin{abstract}
- Additional data are published online only. To view these files please visit the journal online at (http://ard.bmj.com).

${ }^{1} \mathrm{RHIO}$ (Rheumatology, Immunology, Osteology) Center Duesseldorf and RHIO Research Institute, Duesseldorf, Germany ${ }^{2}$ Department of Rheumatology and Clinical Immunology, Charité-University Medicine Berlin, Berlin, Germany ${ }^{3}$ mivenion $\mathrm{GmbH}$, Berlin, Germany

${ }^{4}$ Department of Radiology, Evangelisches Krankenhaus Duesseldorf, Duesseldorf, Germany

${ }^{5}$ SCOSSIS Statistical Consulting, Berlin, Germany
\end{abstract}

\section{Correspondence to}

Gerd R Burmester, Department of Rheumatology and Clinical Immunology, Charité-University Medicine Berlin, Charitéplatz 1 . D-10117 Berlin, Germany: gerd.burmester@charite.de

$\mathrm{H}$-EL and SO contributed equally

Accepted 24 August 2011 Published Online First 12 October 2011

\section{ABSTRACT \\ Background Indocyanine green (ICG)-enhanced fluorescence optical imaging (FOI) is an established technology for imaging of inflammation in animal models. In experimental models of arthritis, FOl findings corresponded to histologically proven synovitis. This is the first comparative study of FOI with other imaging modalities in humans with arthritis.}

Methods $252 \mathrm{FOl}$ examinations (Xiralite system, mivenion $\mathrm{GmbH}$, Berlin, Germany; ICG bolus of $0.1 \mathrm{mg} / \mathrm{kg} /$ body weight, sequence of 360 images, one image per second) were compared with clinical examination (CE), ultrasonography (US) and MRI of patients with arthritis of the hands.

Results In an FOl sequence, three phases could be distinguished (P1-P3). With MRI as reference, FOI had a sensitivity of $76 \%$ and a specificity of $54 \%$, while the specificity of phase 1 was $94 \%$. FOI had agreement rates up to $88 \%$ versus CE, $64 \%$ versus greyscale US, $88 \%$ versus power Doppler US and 83\% versus MRI, depending on the compared phase and parameter. FOI showed a higher rate of positive results compared to CE, US and MRI. In individual patients, FOI correlated significantly $(p<0.05)$ with disease activity (Disease Activity Score 28, $r=0.41)$, US $(r=0.40)$ and RAMRIS (Rheumatoid Arthritis MRI Score) ( $r=0.56)$. FOI was normal in $97.8 \%$ of joints of controls.

Conclusion ICG-enhanced FOl is a new technology offering sensitive imaging detection of inflammatory changes in subjects with arthritis. FOI was more sensitive than CE and had good agreement with CE, US in power Doppler mode and MRI, while showing more positive results than these. An adequate interpretation of an FOI sequence requires a separate evaluation of all phases. For the detection of synovitis and tenosynovitis, FOl appears to be as informative as 1.5 T MRI and US.

\section{INTRODUCTION}

With recent advances in the management of rheumatic diseases, imaging plays a major role in early diagnosis, estimation of prognosis and evaluation of therapeutic outcome. In rheumatoid arthritis (RA), treat-to-target strategies ${ }^{1}$ and the adequate use of disease-modifying drugs ${ }^{12}$ require sensitive instruments that allow a valid detection of affected joints.

Careful clinical examination (CE) is a prerequisite but may miss subclinical inflammation in early disease as well as in clinical remission under treatment. $^{3-5}$ Conventional radiography is commonly used as an indicator of prognosis and represents the standard outcome measure of disease progression in clinical studies but is displaying the result of previous inflammatory processes rather than presenting current disease activity. MRI is considered the gold standard for imaging of synovitis, and MRI bone marrow oedema has been shown to be the strongest independent predictor of radiographic progression in RA. ${ }^{6}$ However, broader usage of MRI in clinical routine settings may be restricted by workflow considerations, cost and limited availability.

Ultrasonography (US) in greyscale mode (GSUS) and US in power Doppler mode (PDUS) have been demonstrated as valid tools for the assessment of synovitis and scoring of clinical activity in RA. ${ }^{8-10}$ In daily clinical practice, PDUS is more available than MRI and often used for fast and dynamic assessment of joint inflammation. ${ }^{11}$ However, apart from clinical studies, the examination procedure is usually limited to a reduced number of joints ${ }^{12}$ due to time constraints.

Fluorescence optical imaging (FOI) is an established technology that has been evaluated for imaging of inflammation in a variety of animal models. ${ }^{13}$ In experimental models of arthritis, indocyanine green (ICG)-enhanced FOI findings corresponded to histologically proven synovitis. ${ }^{14} 15$ The feasibility of this approach in humans was tested, ${ }^{16} 17$ and an FOI system with fixed optical geometry was developed (Xiralite X4; mivenion GmbH, Berlin, Germany).

We report the results of the first comparative study of this commercially available FOI system with CE, US and contrast-enhanced MRI in two major cohorts of patients with arthritis and allied conditions and controls.

\section{PATIENTS AND METHODS \\ Patients}

Two hundred and fifty-two subjects with arthritis and allied conditions were recruited in two centres. One hundred and fifty-three consecutive FOI examinations were evaluated in centre 1 . Ninetynine outpatients were recruited randomly in centre 2. Inclusion criteria were symptoms in the hands and agreement for participation in the study. Two patients did not want to participate in the study 
after detailed information. Six healthy individuals and six subjects with arthralgia without any sign of an inflammatory rheumatic disease served as the control group.

The study was performed in compliance with the Declaration of Helsinki. The study protocol was approved by the ethics committee of the Charité University Clinic Berlin. All study participants had signed consent forms after receiving written and oral information.

\section{Clinical and laboratory assessment}

$\mathrm{CE}$ and laboratory tests (erythrocyte sedimentation rate (ESR) and $\mathrm{C}$ reactive protein (CRP)) were performed. Clinical swollen and tender joints (including distal interphalangeal joint (DIP)) were scored for presence and absence (0-1). The Disease Activity Score 28 (DAS28) ${ }^{18}$ was used to assess disease activity in patients with RA, psoriatic arthritis (PsA) and undifferentiated arthritis (uA).

\section{Ultrasonography}

Seventy-four subjects from centre 2 were examined by GSUS and PDUS (Mylab 70 XVG, Esaote, Genova, Italy). Nine hundred and sixty-two joints (wrist, metacarpophalangeal joint (MCP) $2-5$, proximal interphalangeal joint (PIP) 2-5 and DIP 2-5 of the clinically dominant hand) were evaluated semiquantitatively (grades 0-3) for synovitis and synovial/tenosynovial vascularity in a standardised manner. ${ }^{912}$ Tenosynovitis was scored for presence and absence (0-1). For individual patients, a US sum score over the evaluated joints was calculated.

\section{Magnetic resonance imaging}

Fat-saturated coronal proton-density (FS-PD-TSE), non-enhanced and enhanced T1-TSE with subtraction, coronal and axial fatsaturated postintravenous gadolinium (Dotarem, $0.2 \mathrm{ml} / \mathrm{kg} /$ body weight) (FS-T1-TSE) sequences of the clinically dominant hand were performed in 25 patients (1.5 T MRI; Siemens Magnetom Symphony, Erlangen, Germany). MRI findings (MCP 1-5, interphalangeal joint finger 1 (IP), PIP 2-5, DIP 5 and wrist as a whole) were scored according to the OMERACT (Outcome Measures in Rheumatoid Arthritis Clinical Trials) criteria. ${ }^{19-21}$ The RAMRI score was calculated.

\section{Fluorescence optical imaging}

Background of the technology and details of the Xiralite system (figure 1) are described in a supplementary text (only online available). The FOI examination follows a standardised procedure: both hands are placed on a preformed hand rest. Ten seconds after starting the examination, an ICG bolus is injected (ICG-Pulsion, $0.1 \mathrm{mg} / \mathrm{kg} /$ body weight intravenously). The system acquires one image every second. Any alteration of fluorophor concentration can be depicted as alteration of signal intensity. In a pilot study, it was found that the signal enhancement had significantly decreased after an examination period of $6 \mathrm{~min}$. Therefore, this duration was determined as the standard examination time.

FOI findings were analysed separately for an individual sequence of 360 images and an electronically generated composite image (CI), automatically obtained by means of the integrated software XiraView (version 3.6). A semiquantitative score was applied for assessment of FOI findings $(0=$ no signal enhancement, $1=$ low $(\leq 25 \%), 2=$ moderate $(>25 \%, \leq 50 \%)$, $3=$ strong ( $>50 \%$ of affected joint area)). For individual patients, a sum score over all joints (DIP 2-5, PIP 2-5, IP, MCP 1-5, wrist and both hands; range 0-90) was calculated. All FOI findings were analysed and reported by one reader.

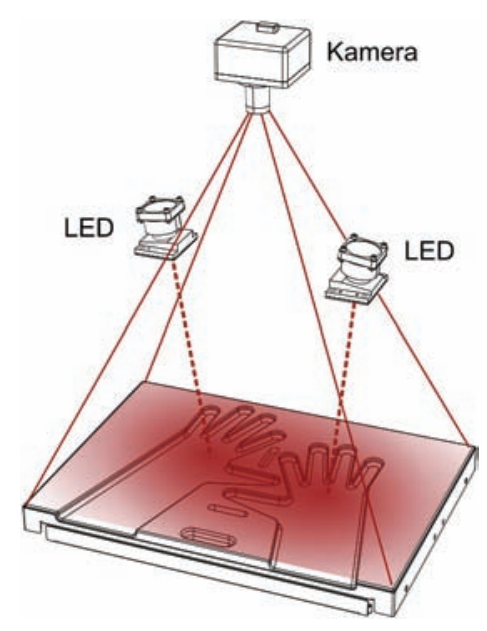

Figure 1 Schematic display of the Xiralite system. A preformed hand rest is used for reliant positioning of both hands. High-power lightemitting diodes (LEDs) illuminate the field of view with dark red light, whereas a specialised digital camera system (charge-coupled device) records the fluorescence signal intensities in the near-infrared spectrum.

\section{Statistical analysis}

Data evaluation and statistical analysis were performed using SAS 9.2 (SAS Institute, Cary, North Carolina, USA). Analyses were conducted at the patient and individual joint level. MRI, GSUS and PDUS were used as the standard reference method for calculation of sensitivity and specificity. The agreement rates (ARs) between pairs of modalities (FOI, CE, US and MRI; swollen and tender joints, synovitis and tenosynovitis), sensitivities and specificities were calculated along with confidence intervals on all joints with non-missing data using a modified adjusted $\chi^{2}$ test $^{22}$ to cover correlations of multiple joints within the same patient. The AR was determined taking into consideration all joints that were affected (grades 1-3) or not affected (grade 0) in both modalities. Comparisons of AR of subgroups (RA, uA and PsA) were performed using appropriate contrasts within logistic regression models. For further analyses of efficacy, summary statistics, frequency counts and $95 \%$ confidence intervals were computed as appropriate. The correlations of the scores were calculated using Spearman's rank correlation coefficient. Statistical significance was concluded with two-sided $\mathrm{p}$ values below 0.05 .

\section{RESULTS}

\section{Patients' characteristics}

The main clinical, laboratory, MRI and US characteristics are detailed in table 1.

\section{Morphological FOI findings}

Analysing a single FOI sequence, we found that, within each image stack, three phases (P1-P3) may be distinguished as defined by the different time points' increased signal intensities in the fingertips. Figure 2 shows a typical FOI image of highly active RA, displaying focal increased signal intensity in all three phases. Inflammatory activity in a variety of affected structures was also detected in PsA (figure 3). A triangular, slightly arcuate enhancement from nail bed into DIP was observed in 60 out of $64(94 \%)$ subjects with PsA compared with 8 out of $38(21 \%)$ in patients with definite RA (sensitivity $94 \%$, specificity $79 \%$, positive predictive value 0.88 , negative predictive value 0.88 ; subjects with both RA and psoriasis have been excluded from the calculation). 
Table 1 Demographic and clinical data of study population

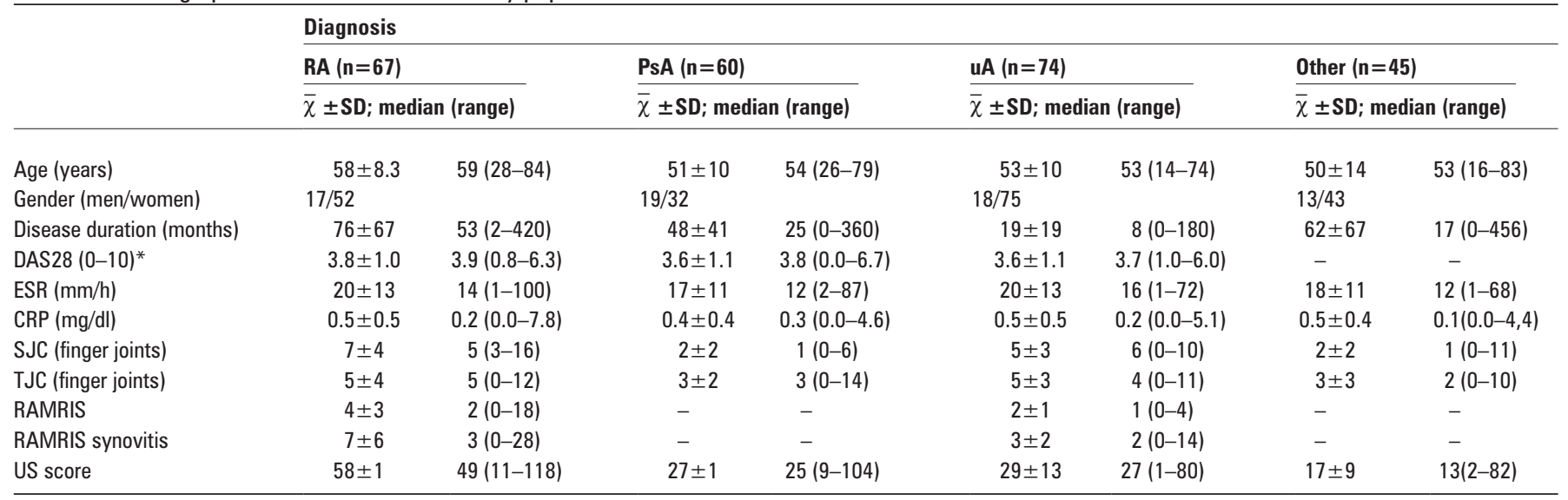

*DAS28 only calculated for RA, PsA and uA.

Other six healthy controls $=$ six arthralgia being above suspicion of inflammatory disease.

CRP, C reactive protein; DAS28, Disease Activity Score 28; ESR, erythrocyte sedimentation rate; PsA, psoriatic arthritis; RA, rheumatoid arthritis; RAMRIS, Rheumatoid Arthritis MRI Score; SJC, swollen joint count; TJC, tender joint count; uA, undifferentiated arthritis; US, ultrasonography.

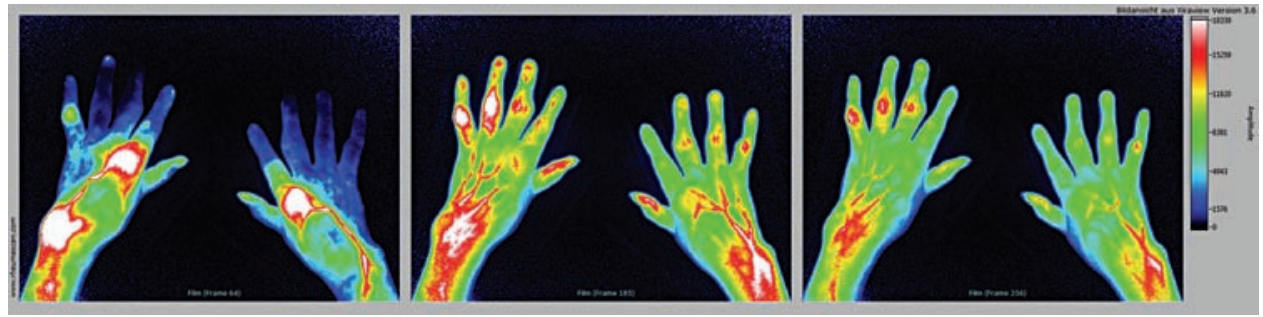

Figure 2 Fol findings in active rheumatoid arthritis (RA). Phase 1 (A) displays focal increases signal intensities until first increased signals in fingertips (early enhancement phase), phase 2 (B) shows increased signal intensities at the same time as signals in fingertips (intermediate phase), and phase $3(C)$ is reached after increased signal intensities of fingertips has decreased (late phase).

\section{Centre 1}

FOI findings were compared with clinical findings in 750 joints (128 tender, 148 swollen, 83 swollen and tender). FOI was compared with MRI findings in 300 joints (figure 4). Fifty-nine joints showed MRI synovitis, and 75 joints showed synovitis or tenosynovitis. MRI detected inflammatory changes in 31 out of 194 $(16 \%)$ clinically asymptomatic joints. FOI displayed positive findings in 387 out of $750(77 \%)$ joints and in 250 out of 557 (45\%) clinically asymptomatic joints.

\section{Sensitivity and specificity}

Taking MRI as the gold standard for inflammatory changes (synovitis or tenosynovitis), CE had a sensitivity of $53 \%$ and a specificity of $81 \%$. FOI had a sensitivity of $76 \%$ and a specificity of $54 \%$. Specificity of phase 1 (P1) and phase 3 (P3) was high $(94 \%$ and $89 \%)$, with corresponding low sensitivity $(27 \%$ and $47 \%$ ) (table 2).

\section{Agreement rates}

AR of $\mathrm{CE}$ and MRI ranged from $63 \%$ to $87 \%$, AR of $\mathrm{CE}$ and $\mathrm{FOI}$ ranged from $44 \%$ to $88 \%$ and $\mathrm{AR}$ of $\mathrm{MRI}$ and FOI ranged from $48 \%$ to $88 \%$, depending on the parameter and subgroup (tables 3,4, S1 and S2). The disagreement mainly resulted from the higher rate of positive findings in FOI. The highest agreement was found for P1, and the lowest was found for phase 2 (P2).

Correlations of FOI with assessments of disease activity

FOI scores correlated significantly and relevantly with the Rheumatoid Arthritis MRI Score (RAMRIS) $(r=0.66, p<0.0001)$ and RAMRIS synovitis ( $\mathrm{r}=0.56, \mathrm{p}<0.0001$ ), weakly correlated with DAS28 $(r=0.32, p<0.0001)$ and did not correlate with laboratory parameters of systemic inflammation (ESR and CRP).

\section{Centre 2}

FOI findings were compared with clinical findings in 1110 joints (244 tender, 261 swollen, 85 tender and swollen). FOI was compared with GSUS and PDUS findings of the clinically dominant hand in 962 joints. Four hundred and fifty-three joints were positive in GSUS, 148 in PDUS and 136 in GSUS and PDUS (including tenosynovitis). A total of 303 out of 522 (58\%) clinically asymptomatic joints showed positive findings in GSUS and 53 out of $522(10 \%)$ showed positive findings in PDUS. FOI displayed positive findings in 864 out of 1110 (78\%) joints and in 473 out of $615(77 \%)$ clinically asymptomatic joints.

\section{Sensitivity and specificity}

Taking GSUS as reference, CE had a sensitivity of $25 \%$ and a specificity of $90 \%$ and FOI had a sensitivity of $70 \%$ and a specificity of $48 \%$. With PDUS as reference, CE had a sensitivity of $43 \%$ and a specificity of $88 \%$ and FOI had a sensitivity of $74 \%$ and a specificity of $42 \%$. Specificity of P1 and P3 were high (GSUS: $95 \%$ and $78 \%$; PDUS: $90 \%$ and $69 \%$ ) with corresponding lower sensitivity (GSUS: $22 \%$ and $51 \%$; PDUS: $33 \%$ and $60 \%$ ) (table 2).

\section{Agreement rates}

ARs of CE and GSUS ranged from $56 \%$ to $60 \%$, ARs of CE and PDUS ranged from $76 \%$ to $84 \%$, ARs of $\mathrm{CE}$ and FOI ranged 


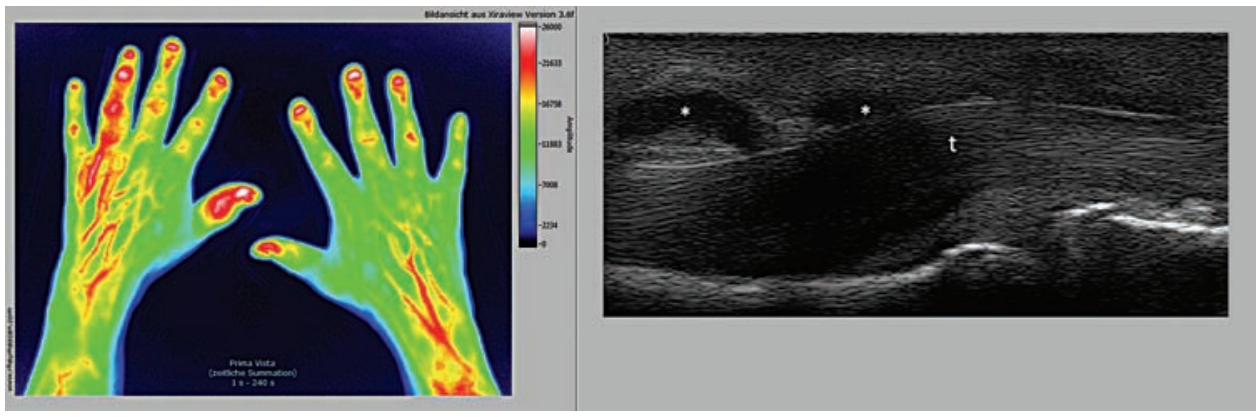

Figure $3 \mathrm{FOI}(\mathrm{Cl})$ and GSUS in psoriatic arthritis (PsA). FOI shows signal intensities in projection of the joints, tendons, osseous and periosteal structures corresponding to clinical dactylitis. Besides, triangular, slightly arcuate enhancements in projection of the nail bed in fingers 3 and 4 of the right hand are seen. GSUS presents an anechoic structure $\left(^{*}\right)$ around the hyperechoic flexor tendon (t) corresponding to tenosynovitis. $\mathrm{Cl}$, composite image; FOI, fluorescence optical imaging; GSUS, ultrasonography in greyscale mode; PsA, psoriatic arthritis.

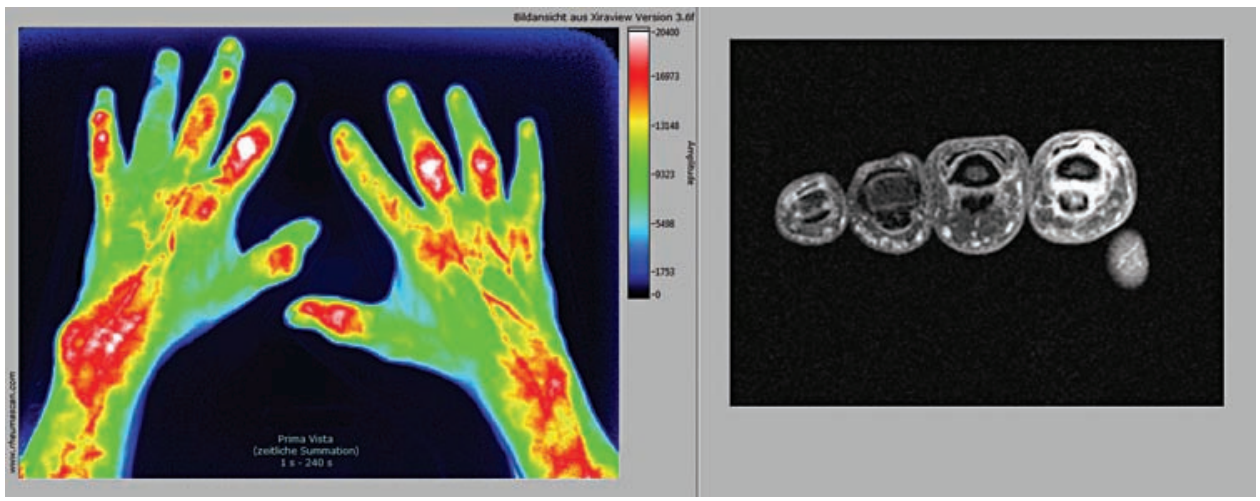

Figure 4 Comparison of $\mathrm{FOI}(\mathrm{CI})$ and $\mathrm{MRI}$ findings. Corresponding signal intensities in FOI and contrast-enhanced (gadolinium) MRI in PIP 2, 3, and 5 of the left hand $(\mathrm{A}, \mathrm{B})$. Cl, composite image; FOI, fluorescence optical imaging.

from $35 \%$ to $88 \%$, ARs of GSUS and FOI ranged from $53 \%$ to $72 \%$ and ARs of PDUS and FOI ranged from $46 \%$ to $82 \%$, depending on the parameter and subgroup (tables 3, 4, S1 and S3). The disagreement mainly resulted from the higher rate of positive findings in FOI. The highest agreement was found for $\mathrm{P} 1$, and the lowest was found for P2.

\section{Correlation of FOI with assessments of disease activity}

FOI scores correlated significantly and relevantly with DAS28 $(\mathrm{r}=0.41, \mathrm{p}<0.0001)$ and US $(\mathrm{r}=0.40, \mathrm{p}=0.0008)$ but not with laboratory parameters (ESR and CRP).

\section{Subgroup analysis}

Generally, the AR did not differ significantly in RA, PsA or uA. Exceptions were the AR for FOI versus CE $(s+t)$ in $R A$ and $u A$ $(p=0.0309)$ and the AR of FOI and GSUS in RA compared to $u A$ $(p=0.0018)$ and PsA $(p=0.0017$, tables S2 and S3).

\section{Control group}

In 12 controls ( 6 healthy and 6 with arthralgia without any sign of inflammatory rheumatic disease; median age 30 years, range $21-56$ years, 3 women), 360 joints were evaluated. FOI did not detect any positive findings in $97.8-100 \%$ of joints (figure S1), depending on the evaluated image or phase. FOI displayed positive findings in 1 out of 360 joints $(0.3 \%)$ in CI, 8 out of 360 joints $(2.2 \%$, grade 1 changes) in P2 and none in P1 and P3. MRI was available in five controls. While MRI was normal, FOI showed minimal changes in CI and P2 (1 out of 60 joints, $1.2 \%$, and 2 out of 60 joints, $3.3 \%$ ) and none in P1 and P3.

\section{Safety}

In all subjects, the procedure was well tolerated. Adverse events were not observed.

\section{DISCUSSION}

ICG-enhanced FOI with the Xiralite system is a new imaging technology. To our knowledge, this is the first study to evaluate the application in patients with arthritis and to compare it with CE, US and MRI.

We found that FOI agreed well with CE, MRI and US. FOI was more sensitive for detecting synovitis and tenosynovitis than CE. FOI showed a higher rate of positive findings than the other compared modalities. In an FOI sequence, three phases could be distinguished, with different sensitivity and specificity as well with different AR. FOI scores correlated significantly with assessment of disease activity (DAS28, US score, RAMRIS). In healthy subjects, FOI was negative in almost all joints.

FOI relies on the fluorescence optical detection of vascularity in inflamed tissues by the means of ICG as a fluorophor. ${ }^{2324}$ Angiogenesis is an early event and is highly dysregulated in inflammatory disorders such as arthritis or psoriasis. ${ }^{25}$ In RA, hypervascularisation and angiogenesis of the synovial membrane are considered to be primary pathogenic mechanisms responsible for the aggressiveness of the rheumatoid pannus on the joint and are suggested as the link to bone destruction. ${ }^{26}$ Synovial vascularisation correlated with the disease activity of a given joint, ${ }^{27} 28$ with radiographic progression ${ }^{29}$ and with the therapeutic response in patients with RA. ${ }^{5}$ 
Table 2 Sensitivity and specificity of FOI and CE versus PDUS, GSUS and MRI (synovitis or tenosynovitis) as standards of reference along with 95\% confidence intervals

\begin{tabular}{|c|c|c|c|c|c|c|}
\hline \multirow[t]{2}{*}{ FOI } & \multicolumn{2}{|l|}{ MRI S or T } & \multicolumn{2}{|l|}{ PDUS } & \multicolumn{2}{|l|}{ GSUS } \\
\hline & Sensitivity & Specificity & Sensitivity & Specificity & Sensitivity & Specificity \\
\hline \multirow[t]{2}{*}{$\mathrm{FOI} \mathrm{Cl}$} & $51 \%(38 / 75)$ & $81 \%(182 / 225)$ & $67 \%(88 / 132)$ & $54 \%(358 / 661)$ & $56 \%(223 / 398)$ & $57 \%(227 / 395)$ \\
\hline & $41 \% ; 60 \%$ & $72 \% ; 89 \%$ & $58 \% ; 76 \%$ & $49 \% ; 59 \%$ & $50 \% ; 62 \%$ & $51 \% ; 64 \%$ \\
\hline \multirow[t]{2}{*}{ F0I P1 } & $27 \%(20 / 75)$ & $94 \%(209 / 223)$ & $33 \%(49 / 148)$ & $90 \%(736 / 814)$ & $22 \%(100 / 453)$ & $95 \%(482 / 509)$ \\
\hline & $10 \% ; 43 \%$ & $89 \% ; 98 \%$ & $21 \% ; 45 \%$ & $87 \% ; 94 \%$ & $15 \% ; 29 \%$ & $92 \% ; 97 \%$ \\
\hline \multirow[t]{2}{*}{ FOI P2 } & $72 \%(54 / 75)$ & $56 \%(127 / 225)$ & $72 \%(106 / 148)$ & $44 \%(361 / 814)$ & $68 \%(306 / 453)$ & $50 \%(256 / 509)$ \\
\hline & $63 \% ; 81 \%$ & $46 \% ; 67 \%$ & $62 \% ; 81 \%$ & $40 \% ; 49 \%$ & $62 \% ; 73 \%$ & $45 \% ; 56 \%$ \\
\hline \multirow[t]{2}{*}{ FOI P3 } & $47 \%(35 / 75)$ & $89 \%(201 / 225)$ & $60 \%(89 / 148)$ & $69 \%(561 / 814)$ & $51 \%(229 / 453)$ & $78 \%(396 / 509)$ \\
\hline & $36 \% ; 58 \%$ & $83 \% ; 95 \%$ & $50 \% ; 71 \%$ & $63 \% ; 75 \%$ & $43 \% ; 58 \%$ & $72 \% ; 84 \%$ \\
\hline \multirow[t]{2}{*}{ Any phase (P1-P3) } & $76 \%(57 / 75)$ & $54 \%(122 / 225)$ & $74 \%(110 / 148)$ & $42 \%(340 / 814)$ & $70 \%(318 / 453)$ & $48 \%(243 / 509)$ \\
\hline & $67 \% ; 85 \%$ & $43 \% ; 65 \%$ & $65 \% ; 84 \%$ & $37 \% ; 47 \%$ & $64 \% ; 76 \%$ & $42 \% ; 53 \%$ \\
\hline CE & $53 \%$ (39\%; 67\%) & $81 \%(76 \% ; 87 \%)$ & $43 \%$ (31\%; 54\%) & $88 \%(83 \% ; 92 \%)$ & $25 \%$ (18\%; 31\%) & $90 \%(86 \% ; 94 \%)$ \\
\hline
\end{tabular}

Any phase, positive in any phase; CE, clinical examination (swollen joints); Cl, composite image; FOl, fluorescence optical imaging; GSUS, ultrasonography in greyscale mode;

$\mathrm{P1}$, phase 1; P2, phase 2; P3, phase 3; PDUS, ultrasonography in power Doppler mode; S, synovitis; T, tenosynovitis.

Table 3 ARs of fluorescence optical imaging with CE, MRI and US

\begin{tabular}{|c|c|c|c|c|c|c|c|c|c|c|c|}
\hline & \multicolumn{2}{|c|}{ CE s (\%) } & \multicolumn{2}{|c|}{ CE t (\%) } & \multicolumn{2}{|c|}{ CE s+t (\%) } & \multirow[b]{2}{*}{ MRI S (\%) } & \multirow[b]{2}{*}{ MRI T (\%) } & \multirow[b]{2}{*}{ MRI S/T (\%) } & \multirow[b]{2}{*}{ GSUS (\%) } & \multirow[b]{2}{*}{ PDUS (\%) } \\
\hline & C1 & C2 & C1 & C2 & C1 & C2 & & & & & \\
\hline $\mathrm{FOI} \mathrm{Cl}$ & 72 & 46 & 71 & 44 & 73 & 44 & 73 & 74 & 73 & 58 & 46 \\
\hline F0I P1 & 79 & 82 & 80 & 80 & 84 & 88 & 78 & 83 & 77 & 60 & 82 \\
\hline FOI P3 & 78 & 70 & 77 & 66 & 81 & 70 & 79 & 79 & 79 & 64 & 68 \\
\hline
\end{tabular}

More detailed tables S1, S2 and S3 including $95 \% \mathrm{Cl}$ are only available online.

$\mathrm{AR}$, agreement rate; $\mathrm{c1}$, centre 1; $\mathrm{c2}$, centre 2; CE, clinical examination; $\mathrm{Cl}$, composite image; FOl, fluorescence optical imaging; GSUS, ultrasonography in greyscale mode;

$\mathrm{P1}$, phase 1; P2, phase 2; P3, phase 3; PDUS, ultrasonography in power Doppler mode; s, swollen joints; $S$, synovitis; $t$, tender joints; $T$, tenosynovitis.

In animal models, FOI has been shown as an appropriate method to identify inflammatory changes in arthritic joints. ${ }^{15} 29$ The histopathological findings of these studies showed early inflammatory changes in FOI-positive joints.

In the present study, FOI was compared to CE, MRI and US in two larger cohorts of patients with arthritis and in healthy controls. The major findings were comparable in both centres.

\section{FOI versus CE}

FOI agreed well with clinically swollen and tender joints. Disagreement of FOI and CE mainly resulted from the higher rate of positive findings in FOI. The highest agreement was seen for FOI P1 and swollen and tender joints, indicating that P1 displays joints with high clinical activity. With MRI or US as reference, FOI was more sensitive than CE.

\section{FOI versus MRI}

FOI agreed well with MRI synovitis and tenosynovitis. Taking MRI as reference, FOI had a sensitivity of $76 \%$ and a specificity of $54 \%$, with a higher specificity $(94 \%$ and $89 \%)$ and a lower sensitivity (27\% and $47 \%$ ) for $\mathrm{P} 1$ and $\mathrm{P} 3$, respectively. AR was up to $88 \%$. Thus, FOI is able to detect MRI synovitis and tenosynovitis reliably. Disagreement of FOI and MRI mainly resulted from the higher rate of positive findings in FOI. A possible explanation is that the different imaging modalities display distinct aspects of the underlying inflammatory pathology. This hypothesis can be proven only by histological examinations.

\section{FOI versus US}

FOI agreed well with US. With GSUS or PDUS as reference, we found a sensitivity of $70 \%$ and $74 \%$ and a specificity of $48 \%$ and $42 \%$, respectively. A higher specificity and a lower sensitivity were seen for P1 (GSUS 95\% and $22 \%$; PDUS $90 \%$ and $33 \%$ ) and P3 (GSUS 78\% and 51\%; PDUS 69\% and 60\%). AR was up to $82 \%$ with PDUS. Thus, FOI displayed US synovitis and tenosynovitis reliably. Similar to MRI, the disagreement of FOI and US mainly resulted from the higher rate of positive findings in FOI. FOI agreed in a higher range with PDUS, which also displays vascularity.

In a comparative study ${ }^{30}$ with MRI as reference, US revealed a sensitivity of $40-70 \%$ for synovitis and an agreement $(73-100 \%)$ comparable to our findings for FOI. Especially for the inflammatory changes, the sensitivity and agreement of US were lower than those for destructive changes. Similar results were obtained in other studies. ${ }^{31} 32$ US and MRI display both morphological changes (eg, pannus, erosions) and dynamic changes (eg, hyperaemia, hypervascularity, hyperperfusion). FOI only displays the dynamic changes. Because of the broader range of dynamic changes compared to morphological changes, we strongly believe that interpretation of findings is more difficult and this may have an influence on sensitivity and AR.

\section{Controls}

In the control group, FOI was normal in $97.8 \%$ joints. None of the 360 joints was FOI positive in P1 or P3. Positive findings were in low grade. This observation supports the interpretation that the disagreement of FOI with MRI and US in the majority of cases did not result from false-positive findings.

\section{Subgroups}

AR did not differ significantly between patients with RA, PsA and $\mathrm{uA}$ in most scenarios. Thus, this finding suggests that FOI is able to detect inflammatory changes independently of the underlying disease. 
Table 4 ARs of CE with MRI, US and fluorescence optical imaging

\begin{tabular}{|c|c|c|c|c|c|c|c|c|c|c|c|c|c|}
\hline & \multirow[b]{2}{*}{ MRI S (\%) } & \multirow[b]{2}{*}{ MRI T (\%) } & \multirow[b]{2}{*}{ MRI S or T (\%) } & \multirow[b]{2}{*}{ GSUS (\%) } & \multirow[b]{2}{*}{ PDUS (\%) } & \multicolumn{2}{|c|}{ FOI Cl (\%) } & \multicolumn{2}{|c|}{ F0I P1 (\%) } & \multicolumn{2}{|c|}{ F0I P2 (\%) } & \multicolumn{2}{|c|}{ F0I P3 (\%) } \\
\hline & & & & & & C1 & C2 & C1 & C2 & C1 & C2 & C1 & C2 \\
\hline CE s & 76 & 76 & 74 & 60 & 80 & 72 & 46 & 79 & 82 & 58 & 48 & 78 & 70 \\
\hline $\mathrm{CE} \mathrm{s}+\mathrm{t}$ & 81 & 83 & 77 & 56 & 84 & 73 & 88 & 84 & 88 & 56 & 46 & 81 & 70 \\
\hline
\end{tabular}

More detailed tables S1, S2 and S3 including $95 \% \mathrm{Cl}$ are only available online.

$\mathrm{AR}$, agreement rate; $\mathrm{C} 1$, centre 1; $\mathrm{C} 2$, centre 2; CE, clinical examination; $\mathrm{Cl}$, composite image; FOl, fluorescence optical imaging; GSUS, ultrasonography in greyscale mode; P1, phase 1; P2, phase 2; P3, phase 3; PDUS, ultrasonography in power Doppler mode; s, swollen joints; $S$, synovitis; t, tender joints; T, tenosynovitis.

\section{Phases}

AR of FOI and CE, MRI and US differed for the CI and the phases. FOI P1 showed the highest agreement with $\mathrm{CE}$ and PDUS, which suggests that P1 displays high local disease activity with high vascularity. The specificity of P1 and P3 was high. Thus, findings in these phases seem to be of special interest. The highest sensitivity was seen in P2 and is comparable to the sensitivity of US with MRI as reference reported from other studies. ${ }^{30} 32$ While the meaning of the phases is still unclear, an adequate interpretation of an FOI exam requires a specific reading of all the phases.

\section{Subclinical inflammation}

MRI, GSUS and PDUS showed positive findings in 16\%, 58\% and $10 \%$ of clinically asymptomatic joints, respectively. This is in alignment with results of other studies where MRI and US detected a higher rate of affected joints than CE. ${ }^{30-32}$ In our study, FOI showed positive findings in $45 \%$ of clinically asymptomatic joints, indicating that FOI also detects subclinical inflammation.

\section{Differential diagnostic aspects}

A characteristic pattern of signal distribution was seen in patients with PsA. The morphological aspect indicates an association with the synovio-entheseal complex. ${ }^{33}$ This sign may provide additional information for differential diagnosis but has to be validated with a larger number of patients.

\section{Correlations with scores of disease activity}

We found that FOI correlated significantly with disease activity scores (DAS28, US score, RAMRIS). Monitoring of disease activity and valid assessment of remission, the special target for RA treatment, ${ }^{1}$ are a crucial aspect with respect to the rapidly growing armamentarium of disease-modifying drugs. Our data indicate that FOI may be an additional tool for the assessment of disease activity in arthritic conditions.

\section{Limitations}

We are aware of some limitations concerning the image interpretation and quantification of pathological changes. While the examination procedure itself has been standardised in detail, consistent standards for image adjustment and interpretation are not yet established. In this study, we have chosen a semiquantitative evaluation of FOI findings, comparable to US image interpretation. Generally, the digital technology of the Xiralite system allows an automatic image interpretation and quantitative analysis of image sequences, but appropriate software is not yet available. With a substantial ${ }^{34}$ intrareader $(\kappa=0.73)$ and interreader $(\kappa=0.73)$ agreement (separate study, data not published), our method of image interpretation seems to be reliable.

In conclusion, ICG-enhanced FOI with the Xiralite system is a new imaging technology that allows a sensitive and valid assessment of inflammation in arthritis. FOI was comparable to $1.5 \mathrm{~T} \mathrm{MRI}$ and US in detecting synovitis and tenosynovitis. Thereby, it is a fast and safe imaging screening tool for patients with suspected arthritis. Furthermore, FOI is useful for objectifying treatment response and treatment monitoring. FOI was more sensitive than CE. In addition, FOI could be helpful in the differentiation of nail involvement and arthritis of DIPs in patients with psoriasis and/or PsA. However, further investigations are needed for a comprehensive definition of FOI pathologies, advancement of methodical standards and evaluation of sensitivity to change and prognostic value.

Contributors All authors were involved in drafting the article or revising it critically for important intellectual content, and all authors approved the final version to be published. No medical writer was involved in the preparation of the manuscript. SW had full access to all of the data in the study and takes responsibility for the integrity of the data and the accuracy of the data analysis. Study conception and design: H-EL, MB, SW, SO, MB, MS and GRB. Acquisition of data: SW, SO, H-EL, PS, HB, L-A, BK and MB. Analysis and interpretation of data: SW, H-EL, MB, MB and GRB. Manuscript preparation: H-EL, SW, MB, SO, MB and GRB. Statistical analysis: CS, SW, MB and H-EL.

Funding This study was supported by BMBF project "ArthroMark", subproject no. 7 "Clinical study on Biomarkers and Imaging". One of the technical devices (FOI) was provided via an unrestricted educational grant by Pfizer Company, Berlin, Germany. Statistical analysis was funded by mivenion $\mathrm{GmbH}$, Berlin, Germany.

Competing interests M Schirner and M Bahner are shareholders of mivenion GmbH. Patient consent Obtained.

Ethics approval This study was conducted with the approval of the ethics committee of the Charité University Clinic Berlin.

Provenance and peer review Not commissioned; externally peer reviewed.

\section{REFERENCES}

1. Smolen JS, Aletaha D, Bijlsma JW, et al. Treating rheumatoid arthritis to target: recommendations of an international task force. Ann Rheum Dis 2010;69:631-7.

2. Saag KG, Teng GG, Patkar NM, et al. American College of Rheumatology 2008 recommendations for the use of nonbiologic and biologic disease-modifying antirheumatic drugs in rheumatoid arthritis. Arthritis Rheum 2008;59:762-84.

3. Brown AK, Quinn MA, Karim Z, et al. Presence of significant synovitis in rheumatoid arthritis patients with disease-modifying antirheumatic drug-induced clinical remission: evidence from an imaging study may explain structural progression. Arthritis Rheum 2006;54:3761-73.

4. Brown AK, Conaghan PG, Karim Z, et al. An explanation for the apparent dissociation between clinical remission and continued structural deterioration in rheumatoid arthritis. Arthritis Rheum 2008;58:2958-67.

5. Taylor PC, Steuer A, Gruber J, et al. Comparison of ultrasonographic assessment of synovitis and joint vascularity with radiographic evaluation in a randomized, placebocontrolled study of infliximab therapy in early rheumatoid arthritis. Arthritis Rheum 2004;50:1107-16.

6. McQueen FM, Benton N, Perry D, et al. Bone edema scored on magnetic resonance imaging scans of the dominant carpus at presentation predicts radiographic joint damage of the hands and feet six years later in patients with rheumatoid arthritis. Arthritis Rheum 2003;48:1814-27.

7. Bøyesen $\mathbf{P}$, Haavardsholm EA, van der Heijde D, et al. Prediction of MRI erosive progression: a comparison of modern imaging modalities in early rheumatoid arthritis patients. Ann Rheum Dis 2011;70:176-9.

8. Szkudlarek M, Court-Payen M, Strandberg C, et al. Power Doppler ultrasonography for assessment of synovitis in the metacarpophalangeal joints of patients with rheumatoid arthritis: a comparison with dynamic magnetic resonance imaging. Arthritis Rheum 2001:44:2018-23. 
9. Backhaus M, Burmester GR, Sandrock D, et al. Prospective two year follow up study comparing novel and conventional imaging procedures in patients with arthritic finger joints. Ann Rheum Dis 2002:61:895-904.

10. Klauser A, Frauscher F, Schirmer M, et al. The value of contrast-enhanced color Doppler ultrasound in the detection of vascularization of finger joints in patients with rheumatoid arthritis. Arthritis Rheum 2002;46:647-53.

11. Boesen M, Ellegard K, Boesen L, et al. Ultrasound Doppler Score correlates with OMERACT RAMRIS bone marrow oedema and synovitis score in the wrist joints of patients with rheumatoid arthritis. Ultraschall Med 2011:doi:10.1055/s-0029-1245922

12. Backhaus M, Ohrndorf S, Kellner H, et al. Evaluation of a novel 7-joint ultrasound score in daily rheumatologic practice: a pilot project. Arthritis Rheum 2009:61:1194-201.

13. Gompels LL, Lim NH, Vincent T, et al. In vivo optical imaging in arthritis-an enlightening future? Rheumatology (Oxford) 2010;49:1436-46

14. Fischer T, Gemeinhardt I, Wagner S, et al. Assessment of unspecific near-infrared dyes in laser-induced fluorescence imaging of experimental arthritis. Acad Radiol 2006;13:4-13

15. Meier R, Krug C, Golovko D, et al. Indocyanine green-enhanced imaging of antigeninduced arthritis with an integrated optical imaging/radiography system. Arthritis Rheum 2010;62:2322-7.

16. Ebert B, Berger J, Voigt $J$, et al. Early detection of rheumatoid arthritis in humans by fluorescence imaging. Biomedical Optics, OSA Technical Digest (CD). Optical Society of America, 2008, p. BTuF19.

17. Fischer $\mathbf{T}$, Ebert $\mathrm{B}$, Voigt $\mathrm{J}$, et al. Detection of rheumatoid arthritis using non-specific contrast enhanced fluorescence imaging. Acad Radiol 2010;17:375-81.

18. Prevoo ML, van't Hof MA, Kuper HH, et al. Modified disease activity scores that include twenty-eight-joint counts. Development and validation in a prospective longitudinal study of patients with rheumatoid arthritis. Arthritis Rheum 1995; 38:44-8.

19. Conaghan P, Bird P, Ejbjerg B, et al. The EULAR-OMERACT rheumatoid arthritis MRI reference image atlas: the metacarpophalangeal joints. Ann Rheum Dis 2005;64(Suppl 1):i11-21.

20. Ejbjerg B, McQueen F, Lassere M, et al. The EULAR-OMERACT rheumatoid arthritis MRI reference image atlas: the wrist joint. Ann Rheum Dis 2005; 64(Suppl 1):i23-47.

21. Ostergaard M, McQueen F, Wiell $\mathrm{C}$, et al. The OMERACT psoriatic arthritis magnetic resonance imaging scoring system (PsAMRIS): definitions of key pathologies, suggested MRI sequences, and preliminary scoring system for PsA Hands. $J$ Rheumatol 2009;36:1816-24.

22. Schwenke C, Busse R. Analysis of differences in proportions from clustered data with multiple measurements in diagnostic studies. Methods Inf Med 2007:46:548-52.

23. Bremer C, Werner S, Langer HE. Assessing activity of rheumatic arthritis with fluorescence optical imaging. Eur Musculoskelet Rev 2009;4:96-100.
24. Eisenblätter M, Höltke C, Persigehl T, et al. Optical techniques for the molecular imaging of angiogenesis. Eur J Nucl Med Mol Imaging 2010;37(Suppl 1):S127-37.

25. Kennedy A, Ng CT, Biniecka M, et al. Angiogenesis and blood vessel stability in inflammatory arthritis. Arthritis Rheum 2010;62:711-21.

26. Pap T, Distler 0. Linking angiogenesis to bone destruction in arthritis. Arthritis Rheum 2005;52:1346-8.

27. Lindblad S, Hedfors E. Intraarticular variation in synovitis. Local macroscopic and microscopic signs of inflammatory activity are significantly correlated. Arthritis Rheum 1985;28:977-86.

28. Ostergaard M, Hansen M, Stoltenberg M, et al. Magnetic resonance imagingdetermined synovial membrane volume as a marker of disease activity and a predictor of progressive joint destruction in the wrists of patients with rheumatoid arthritis. Arthritis Rheum 1999;42:918-29

29. Fukae $\mathbf{J}$, Kon $\mathbf{Y}$, Henmi M, et al. Change of synovial vascularity in a single finger joint assessed by power Doppler sonography correlated with radiographic change in rheumatoid arthritis: comparative study of a novel quantitative score with a semiquantitative score. Arthritis Care Res (Hoboken) 2010;62:657-63.

30. Wiell C, Szkudlarek M, Hasselquist M, et al. Ultrasonography, magnetic resonance imaging, radiography, and clinical assessment of inflammatory and destructive changes in fingers and toes of patients with psoriatic arthritis. Arthritis Res Ther 2007:9:R119.

31. Backhaus M, Kamradt T, Sandrock D, et al. Arthritis of the finger joints: a comprehensive approach comparing conventional radiography, scintigraphy, ultrasound, and contrast-enhanced magnetic resonance imaging. Arthritis Rheum 1999:42:1232-45.

32. Szkudlarek M, Klarlund M, Narvestad E, et al. Ultrasonography of the metacarpophalangeal and proximal interphalangeal joints in rheumatoid arthritis: a comparison with magnetic resonance imaging, conventional radiography and clinical examination. Arthritis Res Ther 2006;8:R52.

33. McGonagle D, Lories RJ, Tan AL, et al. The concept of a "synovio-entheseal complex" and its implications for understanding joint inflammation and damage in psoriatic arthritis and beyond. Arthritis Rheum 2007:56:2482-91.

34. Landis JR, Koch GG. The measurement of observer agreement for categorical data. Biometrics 1977;33:159-74.

35. Cherrick GR, Stein SW, Leevy CM, et al. Indocyanine green: observations on its physical properties, plasma decay, and hepatic extraction. J Clin Invest 1960;39:592-600.

36. Mordon S, Devoisselle JM, Soulie-Begu S, et al. Indocyanine green: physicochemical factors affecting its fluorescence in vivo. Microvasc Res 1998;55:146-52

37. Haruna M, Kumon K, Yahagi N, et al. Blood volume measurement at the bedside using ICG pulse spectrophotometry. Anesthesiology 1998;89:1322-8.

38. Desmettre T, Devoisselle JM, Mordon S. Fluorescence properties and metabolic features of indocyanine green (ICG) as related to angiography. Surv Ophthalmol 2000;45:15-27. 


\section{Corrections}

Werner SG, Langer H-E, Ohrndorf S, et al. Inflammation assessment in patients with arthritis using a novel in vivo fluorescence optical imaging technology. Ann Rheum Dis 2012;71:504-510. This was published in the print issue with the incorrect doi 10.1136/annrheumdis-2010-148288. The correct doi is 10.1136/ard.2010.148288 because this is the doi it was originally published with online first.

Ann Rheum Dis 2012;11:1756. doi:10.1136/ard.2010.148288 